say that the machine will either perform activities requiring no 'judgment', or it may be adapted to include activities requiring 'judgment' provided that the programme contains some means for evaluating consequences. We can then go further and consider problems which are rather inexact, or poorly defined, with the condition that the answer(s) to the problem (which may be very difficult to find) must be easy to check or recognize as correct when arrived at. In order to do this, the machine changes its own programme in some successive manner and at the same time evaluates the effect of these changes. It certainly appears that this technique of 'machine search' can be regarded as a learning process (perhaps even more ?) in that the machine itself attempts to arrive at the correct way to tackle a problem.

Dr. F. L. MeNaughton (Montreal Neurological Institute) made some remarks on stability in relation to the human system, pointing out first that we are still far from an adequate understanding of how the brain works. Experiments in which people were isolated from their environment result in hallucinations in the experimental subject and some distortion of perception when the period of isolation is ended; a clear interdependence of nervous system and environment is indicated. When this adjustment of man to his enviromment breaks down he shows the symptoms of disease. Broadly speaking, it appears that only damage or disease in the general receptor or motor areas of the brain produce immediately identifiable external symptoms. Considerable parts of other areas of the brain may be removed or quite drastic surgery carried out, with what appear to be only transient after-effects in many cases. In discussion, the close analogy between the apparent organization of the human brain and the problem of 'machine search', mentioned above, was pointed out. It has been found that in machine 'learning' of this type, the initial and final stages of the programme (which might perhaps be likened to receptor and motor areas) are critical in the solution of the problem, but otherwise many alternative programmes appear to be possible.

Dr. G. Glinski (Department of Electrical Engineering, University of Ottawa) spoke on the general problem of stability and adaptability of multi-loop feedback control systems. Systems of this type are much closer to living systems than the simpler control systems discussed earlier since a number of variables are involved in controlling a single output. In addition, there may be feedback to one or more inputs and it is also possible to take into consideration some interaction between input variables.

In the previous symposium, Dr. A. C. Burton (University of Western Ontario) presented some demonstrations on the significance of visual perception. The present symposium concluded with a short demonstration by Dr. Burton of interaction between visual inputs. This followed an experiment first performed by Mach in which an interaction between neighbouring receptors in the eye leading to a sharpening of the contrast between light and dark regions is shown quite dramatically by the apparent presence of intensified dark and light bands in the penumbra region of a straight edge when projected on to a lantern screen.

Following the meeting, a panel discussion took place on the Canadian Broadcasting Corporation system under the chairmanship of Sir Robert WatsonWatt, in which Drs. Burgen, Dunham and MacDonald took part. During this spontaneous discussion the significance of 'learning' by machines was explored further. It is probable that a further symposium will be held--perhaps this time in Montreal.

D. K. C. MacDonald

Dougras L. Martin

\title{
THERMONUCLEAR PROCESSES
}

\begin{abstract}
A CONVENTION on "Thermonuclear Processes" was held in the Great Hall of the Institution of Civil Engineers on April 29-30. The convention was organized by the Institution of Electrical Engineers in conjunction with the British Nuclear Energy Conference. A total of twenty-two papers were read, half of which were concerned exclusively with the engineering problems arising out of research into controlled thermonuclear reactions. The remaining papers were largely re-statements of results given at the Geneva Conference on the Peaceful Uses of Atomic Energy last year, albeit presented in a form more suitable for a predominantly engineering audience. However, some new results were given, particularly in the papers submitted by the United States and the U.S.S.R.
\end{abstract}

The mechanism of energy loss remains the outstanding question in connexion with the toroidal stabilized pinch or Zeta-type discharge. At the Geneva Conference, Dr. S. A. Colgate of the University of California claimed that the entire energy loss during the first 7-8 $\mu$ sec. of the discharge life-time in his small torus was due to runaway electrons with an energy of about $2 \mathrm{keV}$. striking the walls. Later, Drs. Hughes and Kaufmann of Associated Electrical Industries, Ltd., Aldermaston, published results obtained with the Sceptre apparatus showing that impurity ions were drifting around the torus with the unexpectedly high velocity of $10^{8} \mathrm{~cm}$./sec. The angular momentum of the deuterium plasma deduced from these measurements was in agreement with the notion of runaway electrons as the chief source of loss. This happy unanimity was destroyed by the release of two further results during questions at the Convention. First, a search had been made on Zeta for the Doppler shift in impurity spectra indicating drift motion and none had been found. Secondly, the group at Los Alamos under Dr. J. A. Phillips working with Perhapsatron $S 4$ had some evidence to show that the whole of the energy loss from their torus was due to radiation in the vacuum ultra-violet region. Clearly more work will have to be done before the problem is finally resolved.

Mr. G. B. F. Niblett of the Atomic Weapons Research Establishment, Aldermaston, described some new experiments on the rapid compression of plasma. In this work a fast-rising axial magnetic field is created inside a linear discharge tube by means of a long single-turn copper coil coupled to a condenser bank. The resulting ring discharge ionizes the gas and is rapidly collapsed by the rising magnetic pressure. The heating principle is the same as that of the fast linear pinch first reported by Kurchatov at Harwell in 1956, but the changed geometry avoids 
electrode effects and is expected to be hydromagnetically more stable. A ringing discharge with a frequency of about $200 \mathrm{kc} . / \mathrm{s}$. was produced in a $1 \frac{1}{2}$-in. bore tube. A rotating-mirror photograph of the discharge in nitrogen showed successive contractions of the discharge corresponding to the oscillations of the coil current. In other measurements, the radial oscillation of the hollow cylindrical shell of plasma when confined between two magnetic fields was observed. It was suggested that these oscillations might be used to heat the plasma.

A paper presented by Mr. J. D. Jukes of the Atomic Energy Research Establishment, Harwell, analysed a system for extracting electrical energy directly from any fusion reactor which uses the principle of magnetic confinement. To extract the energy, the plasma is taken through a compression cycle by varying the confining magnetic field. During the compressed state, energy accumulates in the plasma due to the burning of the nuclear fuel. When expanded the plasma temperature is low so that fusion reactions occur infrequently and the plasma cools, losing energy by bremsstrahlung radiation. In this way, waste heat is removed from the plasma and a elosed Carnot cycle can be achieved. Since the neutrons produced in the fusion reactions are unaffected by the magnetic field, only the energy released in charged particles can be extracted in this way. Mr. Jukes concluded that it should be possible to withdraw about one-third of the available energy from proposed fusion devices directly as electrical energy.

Dr. C. M. van Atta of the University of California Radiation Laboratory presented a survey of the very large American programme in this field which costs 40 million dollars a year and employs three hundred professional staff. A new experiment demonstrating the propagation of torsional Alfvén waves through plasma was described. To produce the plasma a linear discharge carrying a current of $20 \mathrm{k} . \mathrm{amp}$. in an axial magnetic field of 10 kilogauss is used. The waves are generated by applying an alternating radial electric field between one of the electrodes and the conducting wall of the tube. The measured velocity of propagation increases linearly with the axial magnetic field strength and is in close agreement with theory if the ion density is assumed to correspond to all the gas initially in the tube being ionized. This work is being done by Dr. J. Wilcox at the Radiation Laboratory, Berkeley. Among other experiments reported in this paper were hydrodynamic stability studies by Dr. S. A. Colgate using high current pulses through sodium, and similar studies by Dr. H. P. Furth using plasma and a variety of magnetic field configurations.

An important consideration in the magnetic-mirror method of confinement is the accuracy of the assumption that the magnetic moment of a spiralling charged particle is a constant. The principle of mirror confinement is based on this adiabatic invariance, and since a confined particle may undergo many millions of reflexions from the mirrors during its containment, the accuracy required is of a high order. In experiments by Dr. E. Lauer of Berkeley, positrons with energies of the order $1 \mathrm{MeV}$. resulting from the $\beta$-decay of radioactive neon were shown to be contained between magnetic mirrors for $10^{8}$ reflexions. The distance between the mirrors was $1 \mathrm{~m}$. and the Larmor radius of the electrons $6 \mathrm{~cm}$.

Dr. I. N. Golovin of the Institute of Atomic Energy, Moscow, gave an account of research into magnetic-mirror systems in the Soviet Union. $\mathrm{He}$ described an experiment on the adiabatic invariance of the magnetic moment similar in principle to that reported by Dr. van Atta. In this case electrons from the radioactive decay of tritium were used and confinement for up to $10^{7}$ reflexions had been demonstrated. Work had also been done to find the effect of azimuthal asymmetries in the magnetic field on the number of reflexions. It was found that until the azimuthal variations in the magnetic field strength exceeded about 10 per cent there was no effect on this containment. The remainder of Dr. Golovin's paper was devoted to a discussion of the work on Ogra, the large Russian mirror machine. He stated that Ogra was built to study plasma instabilities and was designed to produce a hot plasma with a density of $10^{12}$ ions $/ \mathrm{cm} .^{3}$ if no instabilities were encountered. The principle of injection in this machine is that of dissociation of the input beam of $200-\mathrm{keV}$. molecular ions through collisions with the residual neutral gas in the volume. By balancing the favourable process of dissociation against the unfavourable one of charge exchange, it can be shown that a dense plasma will only be formed if the input beam current exceeds a certain value, the so-called burn-out current. The burn-out current for Ogra was ealculated assuming that the background neutral gas density was determined by the ionization of neutral gas by fast ions and by the release of neutral gas from the walls through the impact of fast particles. A figure of the order $200 \mathrm{~m}$.amp. was obtained for the formation of a hydrogen plasma. Another assump. tion in the calculation was that the path-length of an injected molecular ion moving in the magnetic field and failing to make a collision should be not less than $10^{5} \mathrm{em}$. before it returns to the injector. This length depends critically on the magnetic field configuration and the ion gun alignment, and one of the first tasks with the machine will be to optimize these. A very low initial pressure is also required and to date the lowest value achieved has been $\sim 3 \times 10^{-8} \mathrm{~mm}$. mercury. In view of the size of the vacuum chamber, $\mathrm{I} .5 \mathrm{~m}$. bore and $20 \mathrm{~m}$. long, this represents a considerable technical achievement. The present ionsource gives $150 \mathrm{~m}$.amp. of molecular ions at 100 $\mathrm{keV}$. Up to the moment only a $30-\mathrm{m}$.amp. beam of ions has been injected into the machine and the burnout condition has not been achieved. Impressive photographs were shown of the beam spiralling in the magnetic field.

During question time, Dr. K. W. Allen of the Atomic Weapons Research Establishment, Aldermaston, raised the subject of the order of magnitude difference in the relevant charge exchange cross-sections as measured by Fedorenko in the U.S.S.R. and by Barnett in the United States. Replying, both Dr. van Atta and Dr. Golovin declared their faith in the measurements carried out in their respective countries, but Dr. van Atta hoped that the work would soon be repeated in a third and preferably neutral country. Other points raised during discussion periods concerned the importance of the spectral region $10 \mathrm{~A}$.$400 \mathrm{~A}$. for plasma measurements, the power lost by synchrotron radiation from the electrons in a magnetically confined plasma and the need for more work on surface bombardment effects.

Finally, Sir George Thomson called for more refined experiments, with purer plasma and more homogeneous magnetic fields. $\mathrm{He}$ concluded by declaring his faith in the ultimate solution of the problem of obtaining economic power from fusion reactions.
R. BICkERTON 